\title{
Maternal obesity accelerated non-alcoholic fatty liver disease in offspring mice by reducing autophagy
}

\author{
SHUGUANG HAN ${ }^{1,2 *}$, FENG ZHU ${ }^{1,3 *}$, XIAOXIA HUANG ${ }^{3,4}$, PANPAN YAN $^{1,3}$, KE XU $^{3}$, \\ FANGFANG SHEN ${ }^{3}$, JIAWEN SUN ${ }^{1}$, ZEYU YANG ${ }^{1}$, GUOXI JIN ${ }^{1,2}$ and YIQUN TENG ${ }^{1,3}$ \\ ${ }^{1}$ Graduate School, Bengbu Medical College; ${ }^{2}$ Department of Endocrinology, The First Affiliated Hospital of \\ Bengbu Medical College, Bengbu, Anhui 233000; ${ }^{3}$ Department of Pediatrics, The Second Affiliated Hospital of \\ Jiaxing University, Jiaxing Second Hospital, Jiaxing, Zhejiang 314000; ${ }^{4}$ The Second Clinical Medical College \\ of Zhejiang Chinese Medical University, Hangzhou, Zhejiang 310000, P.R. China
}

Received November 18, 2020; Accepted March 2, 2021

DOI: $10.3892 /$ etm.2021.10148

\begin{abstract}
Non-alcoholic fatty liver disease (NAFLD) is a chronic liver disease characterized by an excessive accumulation of triacylglycerol in the liver. Autophagy is a lysosome-dependent degradation product recovery process, which widely occurs in eukaryotic cells, responsible for the vital maintenance of cellular energy balance. Previously published studies have demonstrated that autophagy is closely related to NAFLD occurrence and maternal obesity increases the susceptibility of offspring to non-alcoholic fatty liver disease, however, the underlying mechanism of this remains unclear. In the present study, NAFLD mouse models (offspring of an obese mother mouse via high-fat feeding) were generated, and the physiological indices of
\end{abstract}

Correspondence to: Professor Yiqun Teng, Department of Pediatrics, The Second Affiliated Hospital of Jiaxing University, Jiaxing Second Hospital, 1518 Huancheng North Road, Jiaxing, Zhejiang 314000, P.R. China

E-mail: rtr656692@163.com

Dr Guoxi Jin, Department of Endocrinology, The First Affiliated Hospital of Bengbu Medical College, 2600 Donghai Avenue, Bengbu, Anhui 233000, P.R. China

E-mail: jyzjyz1999@163.com

${ }^{*}$ Contributed equally

Abbreviations: NAFLD, non-alcoholic fatty liver disease; AMPK, adenosine 5'-monophosphate (AMP)-activated protein kinase; mTOR, mammalian target of rapamycin; OM, obese mother; CM, chow mother; OM-O, offspring of obese mother; CM-O, offspring of chow mother; TC, total cholesterol; TG, triglycerides; HDL, high-density lipoprotein cholesterol; LDL, low-density lipoprotein cholesterol; IL-6, interleukin-6; $\alpha$-sma, alpha-smooth muscle actin; TGF- $\beta 1$, transforming growth factor beta1; SD rat, Sprague Dawley rat

Key words: maternal obesity, non-alcoholic fatty liver disease, autophagy, AMPK/mTOR the liver were observed using total cholesterol, triglyceride, high-density lipoprotein and low-density lipoprotein serum assay kits. The morphological changes of the liver were also observed via $\mathrm{HE}$, Masson and oil red $\mathrm{O}$ staining. Reverse transcription-quantitative-PCR and western blotting were performed to detect changes of autophagy-related genes in liver or fibrosis marker proteins $(\alpha$-smooth muscle actin or TGF- $\left.\beta_{1}\right)$. Changes in serum inflammatory cytokine IL- 6 levels were determined via ELISA. The results of the present study demonstrated that the offspring of an obese mother were more likely to develop NALFD than the offspring of a chow-fed mother, due to their increased association with liver fibrosis. When feeding continued to 17 weeks, the worst cases of NAFLD were observed and the level of autophagy decreased significantly compared with the offspring of a normal weight mouse. In addition, after 17 weeks of feeding, compared with the offspring of a chow-fed mother, the offspring of an obese mouse mother had reduced adenosine 5'-monophosphate (AMP)-activated protein kinase (AMPK) phosphorylation levels and increased mammalian target of rapamycin (mTOR) phosphorylation levels. These results suggested that a reduced level of AMPK/mTOR mediated autophagy may be of vital importance for the increased susceptibility of offspring to NAFLD caused by maternal obesity. In conclusion, the current study provided a new direction for the treatment of NAFLD in offspring caused by maternal obesity.

\section{Introduction}

Non-alcoholic fatty liver disease (NAFLD) is a multisystem disease that increases the risk of type 2 diabetes, cardiovascular disease and heart disease (1). NAFLD can range from simple steatosis to steatohepatitis and can develop into cirrhosis and subsequently liver cancer in certain cases. NAFLD is endemic worldwide, with a prevalence of $25 \%$ in the Asian population. In 2016, the number of NAFLD cases in the United States was 85.3 million $(2,3)$. A previous study has suggested that maternal obesity is closely related to the occurrence of NAFLD in the offspring. Ayonrinde et al (4) conducted a cohort study on 1,170 17-year-old adolescent mothers and found that maternal obesity and weight gain 
in early to mid-term pregnancy increased the incidence of NAFLD in their babies. A prospective study by Patel et al (5) of 14,541 pregnant women revealed that the higher the body mass index of the mother prior to pregnancy, the greater the probability of NAFLD in their offspring. Hence, it is very important to understand how maternal obesity promotes the formation of NAFLD in offspring.

Some studies have suggested that maternal obesity is related to the onset of NAFLD in the offspring and the oxidation of their lipids $(6,7)$. Previous articles have commonly used male rats that were fed a high-fat diet to establish NAFLD models $(8,9)$. Borengasser et al (10) induced obesity in female Spring Dawley rats (SD) via a high fat diet. Tests on their male offspring demonstrated that the liver energy of the male offspring was reduced and mitochondrial function was damaged; additionally, maternal obesity also reduced the level of fatty acid oxidation in the offspring. Additionally the physical state of the mother,has also been observed to induce NAFLD changes in offspring. Wankhade et al (11) fed C57BL/6J female mice a high-fat diet and extracted DNA from their liver tissue for quantitative analysis and found that high-fat diet resulted in DNA methylation in the offspring leading to NAFLD. Either insulin resistance and changes in intestinal flora may be responsible for maternal obesity and the occurrence of NAFLD in offspring $(12,13)$. The mechanism behind maternal obesity and the development of NAFLD in offspring remains unknown.

Autophagy is a catabolic process inherent to eukaryotic cells, via which cells carry damaged organelles, misfolded proteins or pathogens into the lysosome for degradation in order to maintain cell homeostasis (14). Autophagy serves a vital role in maintaining a balance in cell energy metabolism, controlling organelle quality and the cellular stress response (15). Some studies have shown that liver transcription factor EB can induce liver cell autophagy by regulating the expression of lysosome and autophagy-related genes, such as LC3-II, hence improving liver steatosis $(16,17)$. It has also been shown that the upregulation of sirtuin-3 may lead to the deacetylation and activation of manganese superoxide dismutase, which may inhibit autophagy after depletion of cellular superoxide and promote the formation of fat (18).

Recent NAFLD studies have focused on comparing a before and after scenario regarding NAFLD occurrence, but to the best of our knowledge there is a lack of studies investigating the relationship between maternal obesity and the susceptibility of their offspring to NAFLD and autophagy $(19,20)$. Hence, the present study generated obese maternal mice via a high-fat diet to create NAFLD models in their offspring to explore the influence of maternal obesity on the susceptibility of NAFLD in the offspring and its underlying molecular mechanisms. The results may provide a new direction for the treatment of NAFLD in offspring caused by maternal obesity.

\section{Materials and methods}

Ethics approval. Animal experiments performed in the present study comply with the relevant provisions of the Regulations of the People's Republic of China on the Administration of Experimental Animals and have been reviewed and approved by the Animal Experiments and Animal Experiment Ethics
Committee of The Second Affiliated Hospital of Jiaxing University, Jiaxing Second Hospital (Jiaxing, China; approval no. jxey2017002).

Animals. The obesity model of maternal mice was established for 4 weeks and the gestation period was 3 weeks. Offspring were weaned for 17 weeks after 4 weeks of lactation. A total of 20, 4-week-old C57BL/6J female mice were obtained from the Experimental Animal Center of Jiaxing University (Jiaxing, China), where they were housed in specific pathogen free grade conditions. The temperature was kept at $24 \pm 2^{\circ} \mathrm{C}$, a relative humidity of $50 \pm 10 \%$ and $12 / 12 \mathrm{~h}$ light and dark cycle lighting was maintained. Free access to water and food was provided. The mice were randomly divided into 2 groups. One group was fed a high-fat diet [obese mother (OM) group, $\mathrm{n}=10$ ] containing $60.0 \%$ fat, $19.4 \%$ protein, and $20.6 \%$ carbohydrates (TROPHIC Animal Feed High-Tech Co., Ltd.); the other group was fed a normal diet [chow mother (CM), $n=10$ ] containing $4 \%$ fat, $18 \%$ protein, $53 \%$ carbohydrates and $0.07 \%$ cholesterol (SHOOBREE, Synergetic Medical Bioengineering Co., Ltd.). Common male mice (A total of 10 8-week-old mice from Jiaxing University) were mated with the 2 groups of female mice (1 male: 2 female) and their offspring were defined as offspring of obese mother (OM-O) and offspring of obese mother (CM-O). The offspring of the 2 groups were weaned at 4 weeks old. Subsequently, 10 male offspring from the OM-O and CM-O groups were continuously fed a high-fat diet until they were sacrificed at 17 weeks old. Briefly, a sterile transparent glass container with a gauze pad at the bottom was prepared and 2-4 $\mathrm{ml}$ ether (Huadong Medicine Co., Ltd.) was added to the gauze pad, the container was sealed for 10-20 sec to prevent volatilization, a mouse was added to it, the container was resealed and inhalation anesthesia was performed, since mice breathe fast and tetraplegia is thought to be a sign of successful anesthesia. After anesthesia, $1 \mathrm{ml}$ venous blood was collected. Subsequently, all the mice were euthanized by neck dislocation. Finally, blood and liver tissue obtained from the mice were stored at $-80^{\circ} \mathrm{C}$ for further analysis.

Pathological liver examination. At 17 weeks old, the OM-O and CM-O mice were sacrificed, the liver was quickly resected, a small piece of fresh liver tissue was taken, and the liver specimens were fixed with $4 \%$ paraformaldehyde at $4{ }^{\circ} \mathrm{C}$ for $24 \mathrm{~h}$. They were then prepared and embedded in a paraffin block and $4-\mu \mathrm{m}$ thick sections were cut and used for regular hematoxylin-eosin (each $10 \mathrm{~min}$ at room temperature), oil red $\mathrm{O}$ staining ( $8 \mathrm{~min}$ at room temperature) and Masson staining (ponceau staining for $8 \mathrm{~min}$ and aniline blue for $15 \mathrm{~min}$ at room temperature) to analyze liver tissue changes under confocal microscopy (magnification, x200).

Immunohistochemistry. The liver was harvested, and representative sections were fixed in $4 \%$ paraformaldehyde (Huadong Medicine Co., Ltd.) at room temperature for $24 \mathrm{~h}$. Liver sections from OM-O and CM-O mice were then embedded in paraffin and cut into $4 \mu \mathrm{m}$ sections. The paraffin sections were then dewaxed and hydrated at room temperature by successively adding xylene I (15 min), xylene II (15 min), anhydrous ethanol I (5 min) and anhydrous ethanol II (5 min). The slices were subsequently removed and placed in a ventilated 
laboratory to dry and then washed in distilled water. At $100^{\circ} \mathrm{C}$, EDTA antigen repair buffer (PH8.0; cat. no. P0086; Beyotime Institute of Biotechnology) was used for antigen repair. Hydrogen peroxide (3\%) was added and incubated at room temperature for $10 \mathrm{~min}$ to inactivate the activity of endogenous enzymes. Finally, 2\% bovine serum albumin (cat. no. 37520 Thermo Fisher Scientific, Inc.) was added and incubated for $10 \mathrm{~min}$ at room temperature. Primary antibodies (all ABclonal Biotech Co., Ltd.) against alpha-smooth muscle actin ( $\alpha$-sma) (cat. no. A7240), transforming growth factor beta1 (TGF- $\beta 1$ ) (cat. no. A2124), IL-6 (cat. no. A0286) and their corresponding biotinylated secondary antibodies (HRP Goat Anti-Rabbit IgG; cat. no. AS014; ABclonal) were added to the sections. All antibodies were diluted at 1:500. DAB (cat. no. RC062; Shanghai Recordbio Biological Technology Co., Ltd.) color development was performed and the slides coverslipped. Sections were assessed and imaged under confocal microscopy (magnification, x200). Quantitative analysis was then performed using Image $\mathrm{J}$ software $(1.52 \mathrm{~V}$; National Institutes of Health)

Biochemical index detection. Blood samples (1 ml) were collected from the eyeballs of 10 mice from the OM-O and CM-O groups at 17 weeks of age. Whole blood was placed at $4^{\circ} \mathrm{C}$, centrifuged at $2,200 \times \mathrm{g}$ for $10 \mathrm{~min}$ at $4^{\circ} \mathrm{C}$ and the supernatant was collected. Total cholesterol (TC) assay kit (cat. no. A111-1-1; Nanjing Jiancheng Biological Co., Ltd.), triglycerides (TG) assay kit (cat. no. A110-1-1; Nanjing Jiancheng Biological Co., Ltd.), high- density lipoprotein cholesterol (HDL) assay kit (cat.no. A112-1-1; Nanjing Jiancheng Biological Co., Ltd.), low-density lipoprotein cholesterol (LDL) assay kit (cat. no. A113-1-1; Nanjing Jiancheng Biological Co., Ltd.) and interleukin-6 (IL-6) Elisa kit (cat. no. A007-1-2; Nanjing Jiancheng Biological Co., Ltd.) were detected according to the manufacturer's instructions.

Reverse transcription-quantitative $(R T-q) P C R$. Total RNA was extracted from the liver with TRIzol ${ }^{\circledR}$ reagent (Invitrogen; Thermo Fisher Scientific Inc.) and reverse transcribed to cDNA using the HiFiScript gDNA Removal cDNA Synthesis kit (CoWin Biosciences) according to the manufacturer's protocol. The expression of Beclin-1, ATG3, ATG5, ATG12, P62 and LC3B were detected with SYBR Green mix (BioTek China) using the Thermo Fisher-QS3 (Thermo Fisher Scientific Inc.). $\beta$-actin was used as an internal control. The thermocycling conditions were as follows: Pre-denaturation at $94^{\circ} \mathrm{C}$ for $5 \mathrm{~min}$; followed by 40 cycles of denaturation at $94^{\circ} \mathrm{C}$ for $30 \mathrm{sec}$, annealing at $60^{\circ} \mathrm{C}$ for $30 \mathrm{sec}$ and extension at $72^{\circ} \mathrm{C}$ for $30 \mathrm{sec}$. The primer sequences used in this study are summarized in Table I. The relative mRNA expression was measured using the $2^{-\Delta \Delta \mathrm{Cq}}$ method (21).

Western blotting. Total protein was extracted from the liver tissue of OM-O and CM-O mice for western blotting. A protease phosphate inhibitor (cat. no. P1045; Beyotime Institute of Biotechnology) and Phenylmethanesulfonyl fluoride (cat. no. ST506; Beyotime Institute of Biotechnology) was added to RIPA lysis buffer (cat. no. P0013B; Beyotime Institute of Biotechnology) to extract total proteins from the liver tissues of the OM-O and CM-O groups. The bicinchoninic acid (cat. no. P0010S; Beyotime Institute of Biotechnology) protein determination kit was used to measure total protein concentration. The protein samples were adjusted to the same concentration and then mixed with $1 / 4$ volume of protein loading buffer, boiled and denatured. The total protein content $(2 \mu \mathrm{g})$ was then separated by $10 \%$ SDS polyacrylamide gel electrophoresis and transferred to a PVDF membrane. The membrane was blocked using 5\% skimmed milk powder at room temperature for $2 \mathrm{~h}$ and the corresponding antibody stock solution $(1: 1,000)$ was diluted. Primary antibodies (all ABclonal Biotech Co., Ltd.) against Beclin-1 (cat. no. A10101), ATG3 (cat. no. A5809), ATG5 (cat. no. A0203), ATG12 (cat. no. A19610), p62 (cat. no. A7758), LC3B (cat. no. A5618), adenosine 5'-monophosphate (AMP)-activated protein kinase (AMPK) (cat. no. A1229), phosphorylated (p)-AMPK (cat. no. A1229), mammalian target of rapamycin (mTOR) (cat. no. A2445), p-mTOR (cat. no. AP0115) and $\alpha$-sma (cat. no. A7240), TGF- $\beta 1$ (cat. no. A2124), IL-6 (cat. no. A0286) were added and the sections incubated overnight at $4^{\circ} \mathrm{C}$. The following day, the membrane was washed three times in TBST (TBS/0.1\% Tween x10; cat. no. PS103, Epizyme) for $10 \mathrm{~min}$ each time and the horseradish peroxidase-labeled secondary antibody (Goat Anti-Rabbit IgG; cat. no. AS029; ABclonal, $1: 1,000)$ was diluted with $5 \%$ skimmed milk powder and incubated on a shaker for $90 \mathrm{~min}$ at room temperature. After washing the membrane three times in TBST, a luminescent liquid (ECL: cat.no. P0018S, Beyotime) was added and exposed with the Bio-Rad imaging system. ImageJ software (National Institutes of Health; $1.52 \mathrm{~V}$ ) was used to determine the gray value. $\beta$-actin (cat. no. AC038; ABclonal; 1:1,000) or tubulin (cat. no. AC008; ABclonal; 1:1,000) were used as the loading controls and the gray value of the target protein was compared with the gray value of the internal control to determine relative expression levels.

Statistical analysis. Data were presented as means \pm standard error of the mean (SEM). All the experiments were repeated 3 times. An unpaired Student's t-test was used for comparisons between 2 groups. Statistical analyses were performed using IBM SPSS Statistics 22 software (IBM Corp.) ${ }^{*} \mathrm{P}<0.05$ were considered to indicate a statistically significant difference.

\section{Results}

Maternal obesity promotes NAFLD in mouse offspring. In the present study, 4-week-old female C57BL/6J mice $(n=20)$ were randomly selected and 1 group was fed a high-fat diet (OM group, $n=10)$, and the other group was fed a normal diet (CM group $n=10$ ). After 3 weeks of feeding, the weight began to show differences between the 2 groups and feeding was continued for 1 week. After 4 weeks of high-fat feeding, the body weight of the OM group was significantly higher compared with that of the CM group and the average weight of the OM group was greater compared with the $\mathrm{CM}$ group $(\mathrm{P}<0.05$; Fig. 1A). At 8 weeks old, the female mice were mated with ordinary male mice and the offspring named OM-O and CM-O, respectively. The results demonstrated no difference in the body weight between these 2 groups at 4 weeks of weaning. After high-fat feeding for 12 weeks, the body weight of the OM-O group was high, and after 17 weeks, the weight of the OM-O group 
Table I. Sequences of primers used for RT-qPCR.

\begin{tabular}{lll}
\hline Gene & \multicolumn{1}{c}{ Forward $\left(5^{\prime}-3^{\prime}\right)$} & \multicolumn{1}{c}{ Reverse (5'-3') } \\
\hline ATG5 & GCAGAATGACAGATTTGACCAGTTT & GGTTTCCAGCATTGGCTCTATC \\
ATG12 & TCGGTGCTGTGGGAAGAG & GTGCCAACCAAGTAAATGC \\
ATG3 & GAAGAAGATGATGGTGATGGGGG & TTCCTCGTCTTCTTCATCACACA \\
Beclin-1 & GAGTGGAATGAAATCAATGCTGC & TTTCCACCTCTTCTTTGAACTGC \\
LC3 & CCACACCCATCGCTGAC & AAGGTTTCTTGGGAGGCGTAG \\
P62 & TGCTCCACCAGAAGATCCC & CGGCTTCTCTTCCCTCCATGT \\
-actin & CTAGGCACCAGGGTGTGATG & CTCATTGTAGAAGGTGTGGTGC
\end{tabular}

RT-q, reverse transcription-quantitative; LC3B, light chain 3B; ATG, autophagy associated gene.

$\mathbf{A}$

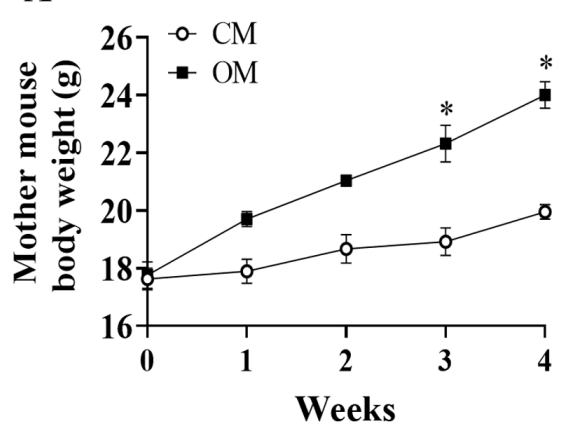

C

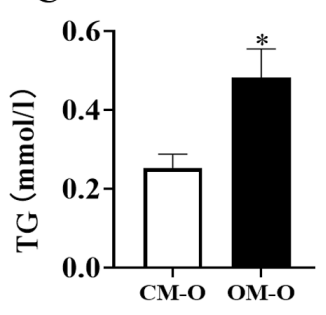

D
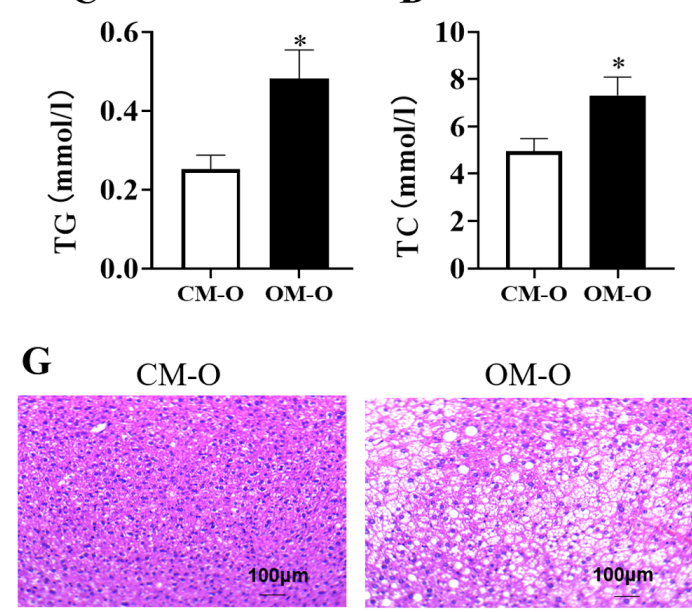

Hematoxylin and eosin
B

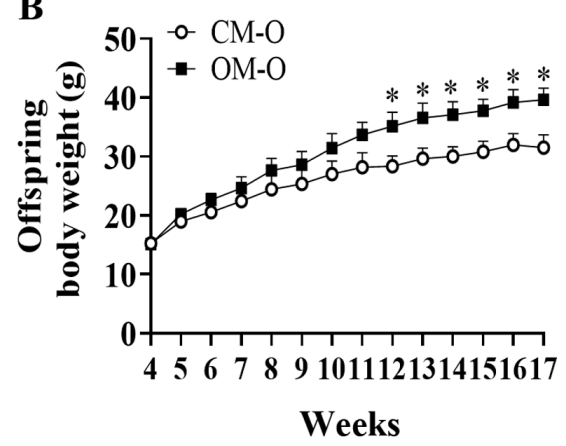

$\mathbf{E}$

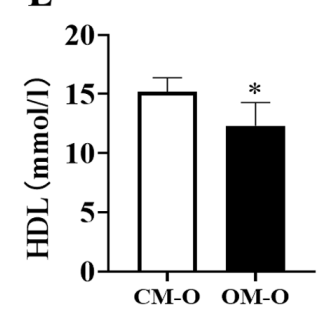

F

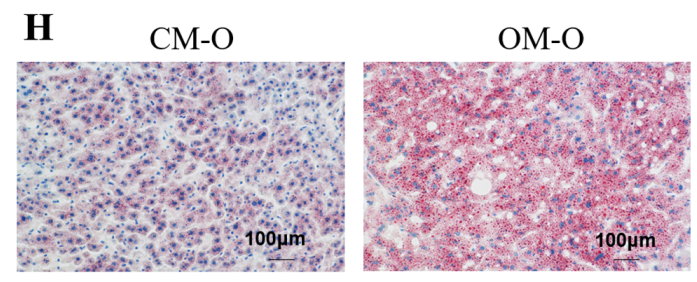

Oil-Red-O

Figure 1. Body weight of mother and NAFLD in mice offspring. (A) Body weight of mouse groups, CM and OM on different diets ( $\mathrm{n}=10 / \mathrm{group}$ ). (B) Body weight of offspring of CM and OM, CM-O and OM-O groups, respectively from 4-17 weeks (n=10/group). (C-F) Serum TG, TC, LDL, HDL at 17 weeks of offspring mice (n=6/group). ( $\mathrm{G}$ and H) Hematoxylin-eosin and Oil-Red-O staining of liver sections from CM-O and OM-O groups at 17 weeks ( $\mathrm{n}=3 / \mathrm{group}$; magnification, $\mathrm{x} 100$ ). Data are shown as mean \pm SEM. ${ }^{*} \mathrm{P}<0.05$. NAFLD, non-alcoholic fatty liver disease; OM, obese mother; CM, chow mother; OM-O, offspring of obese mother; CM-O, offspring of chow mother; TC, total cholesterol; TG, triglycerides; HDL, high-density lipoprotein cholesterol; LDL, low-density lipoprotein cholesterol.

was significantly higher compared with that of the $\mathrm{CM}-\mathrm{O}$ group $(\mathrm{P}<0.05 ;$ Fig. $1 \mathrm{~B})$. In addition, serum TC and TG in the OM-O group were found to be significantly higher compared with the $\mathrm{CM}-\mathrm{O}$ group $(\mathrm{P}<0.05)$, while HDL was significantly lower compared with the CM-O group $(\mathrm{P}<0.05)$, while LDL had an upward trend in the OM-O group compared with the CM-O group (Fig. 1C-F). Following HE staining of offspring liver sections, it was identified that when compared with the liver tissue of the CM-O group, the OM-O group exhibited markedly higher fatty vacuolization (Fig. 1G). Observation of the fat content, according to the degree of redness of the oil red O-stained tissue sections, revealed that the fat content of the OM-O group was significantly higher compared with the CM-O group (Fig. 1H). 
A

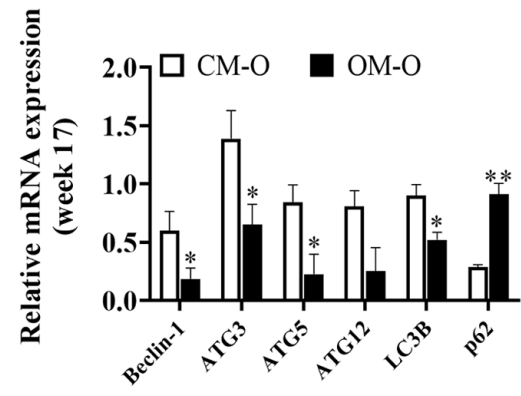

B

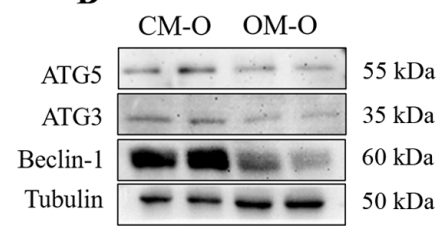

C

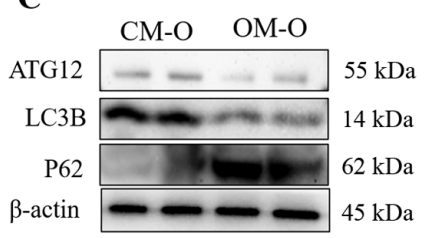

D

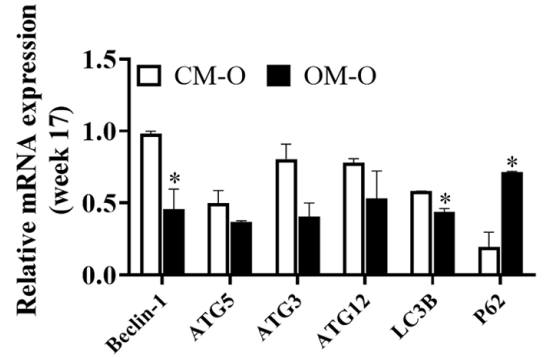

$\mathbf{E}$

CM-O

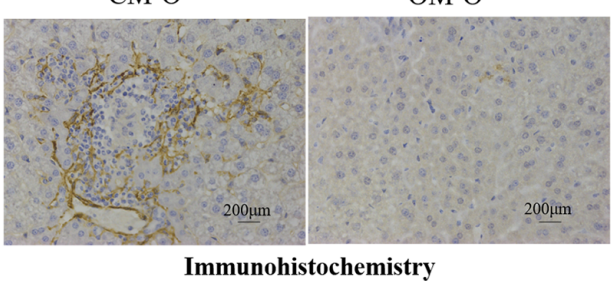

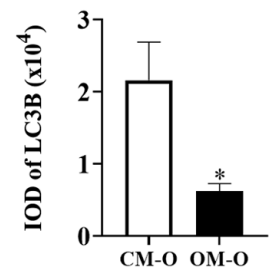

Figure 2. Expression of autophagy-related genes in offspring liver. (A) Expression of genes related to autophagy in 17 weeks old offspring by RT-qPCR ( $n=3 /$ group). (B and C) Western blotting of autophagy-related genes ( $n=3 /$ group). (D) Expression of genes related to autophagy in 17 weeks old offspring by WB ( $\mathrm{n}=3$ /group). (E) Representative picture of LC3B immunohistochemical staining in liver ( $\mathrm{n}=3$ /group; magnification, $\mathrm{x} 200$ ). Data are shown as mean \pm SEM. ${ }^{*} \mathrm{P}<0.05,{ }^{* *} \mathrm{P}<0.01$. OM-O, offspring of obese mother; $\mathrm{CM}-\mathrm{O}$, offspring of chow mother; RT-q, reverse transcription-quantitative; LC3B, light chain 3B; ATG, autophagy associated gene; IOD, integral optical density.

Maternal obesity promotes the decrease of the liver autophagy level in offspring. It was hypothesized that maternal obesity may promote NAFLD in offspring by autophagy regulation. Previous studies have suggested that Beclin-1, Atg3, Atg5 and Atg12 are components of autophagy mechanism, and LC3b is a marker gene of autophagy $(22,23)$. Hence, the present study investigated autophagy related genes by RT-qPCR. At 17 weeks, Beclin-1, ATG3, ATG5, ATG12, and LC3B levels were significantly lower in the OM-O group compared with the CM-O group, while $\mathrm{P} 62$ showed the opposite trend $(\mathrm{P}<0.05)$ (Fig. 2A). It was also revealed that there were significant differences in Beclin-1, LC3B and P62 between the two groups $(\mathrm{P}<0.05)$ (Fig. 2B-D). Furthermore, ATG3, ATG5 and ATG12 levels were decreased. By immunohistochemical detection of the expression level of LC3B, the same result was observed $(\mathrm{P}<0.05)$ (Fig. 2E). These results indicated that the level of autophagy in the liver of the offspring of the obese mice was decreased.

Maternal obesity promotes the decrease of AMPK phosphorylation levels and the increase of mTOR phosphorylation levels in offspring livers. AMPK is a key energy sensor that regulates cellular metabolism to maintain energy homeostasis. Additionally, it is known that autophagy is inhibited by mTOR $(24,25)$. AMPK and its downstream site changes in mTOR phosphorylation were therefore investigated after feeding mice for 17 weeks. The results of showed that the total AMPK and mTOR protein levels remained unchanged in both $\mathrm{CM}-\mathrm{O}$ and OM-O groups. However, compared with CM-O, AMPK phosphorylation levels were decreased and mTOR phosphorylation levels were increased in $\mathrm{OM}-\mathrm{O}(\mathrm{P}<0.05$; Fig. 3). These results indicated that the formation of NAFLD in the offspring of obese female mice may be related to changes in AMPK/mTOR phosphorylation.

Maternal obesity promotes liver fibrosis in offspring mice. Masson staining revealed that the level of fibrosis in the OM-O group was significantly higher compared with $\mathrm{CM}-\mathrm{O}$ group $(\mathrm{P}<0.05$; Fig. 4A). In order to verify the association between the occurrence of NAFLD in the offspring and any changes in liver fibrosis, an ELISA kit was used to determine the serum IL-6 level in the mice offspring at 17 weeks. $\alpha$-sma and TGF- $\beta 1$ lead to liver fibrosis, which further leads to changes in NAFLD. $\alpha$-sma and TGF- $\beta 1$ were chosen as liver fibrosis markers in the present study (26) and western blotting and immunohistochemistry to investigate their expression was performed. The results demonstrated that $\alpha$-sma and TGF- $\beta_{1}$ expression in the OM-O group at 17 weeks were significantly higher compared with the CM-O group ( $\mathrm{P}<0.05$; (Fig. 4B and C). A significant increase in IL-6 levels at 17 weeks was also observed in the OM-O group when compared with the $\mathrm{CM}-\mathrm{O}$ group $(\mathrm{P}<0.05$; Fig. 4D). In addition, the results of western blotting demonstrated that there was significantly higher IL- 6 and TGF- $\beta 1$ 


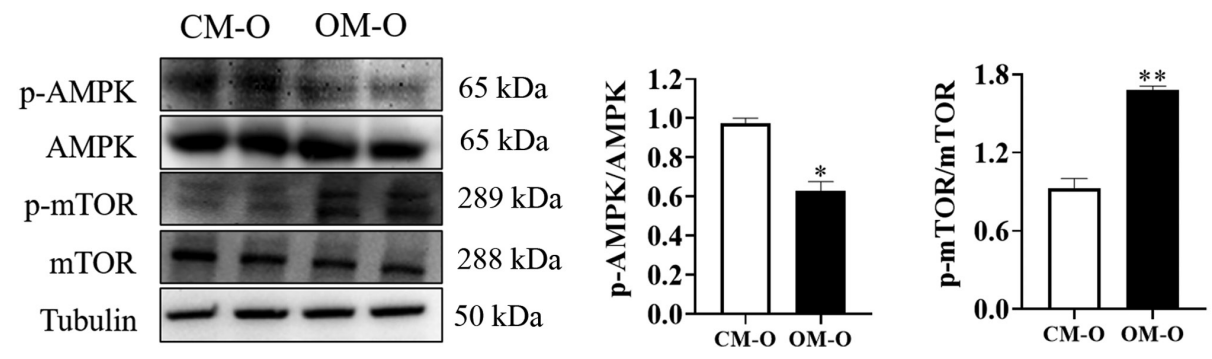

Figure 3. AMPK-mTOR signaling in offspring liver. AMPK, p-AMPK, mTOR and p-mTOR expression was by western blotting. Protein contents were quantified by scanning densitometry of the bands (arbitrary units; $\mathrm{n}=3$ /group). Results are expressed as mean $\pm \mathrm{SEM}$. ${ }^{*} \mathrm{P}<0.05,{ }^{* *} \mathrm{P}<0.01$. OM-O, offspring of obese mother; CM-O, offspring of chow mother; p, phosphorylated; AMPK, adenosine 5'-monophosphate (AMP)-activated protein kinase; mTOR, mammalian target of rapamycin.

A

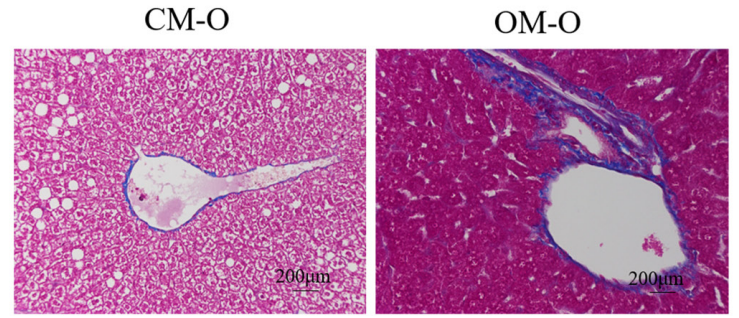

Masson staining

B

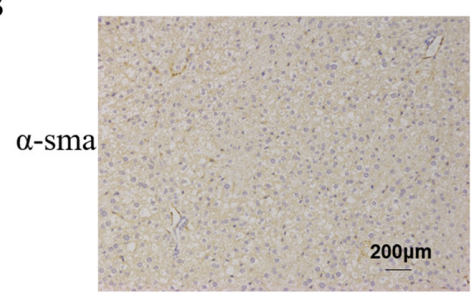

C

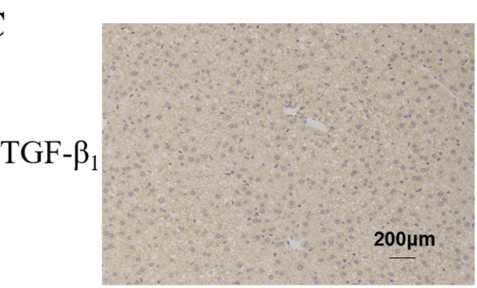

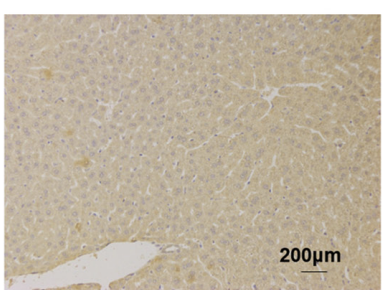
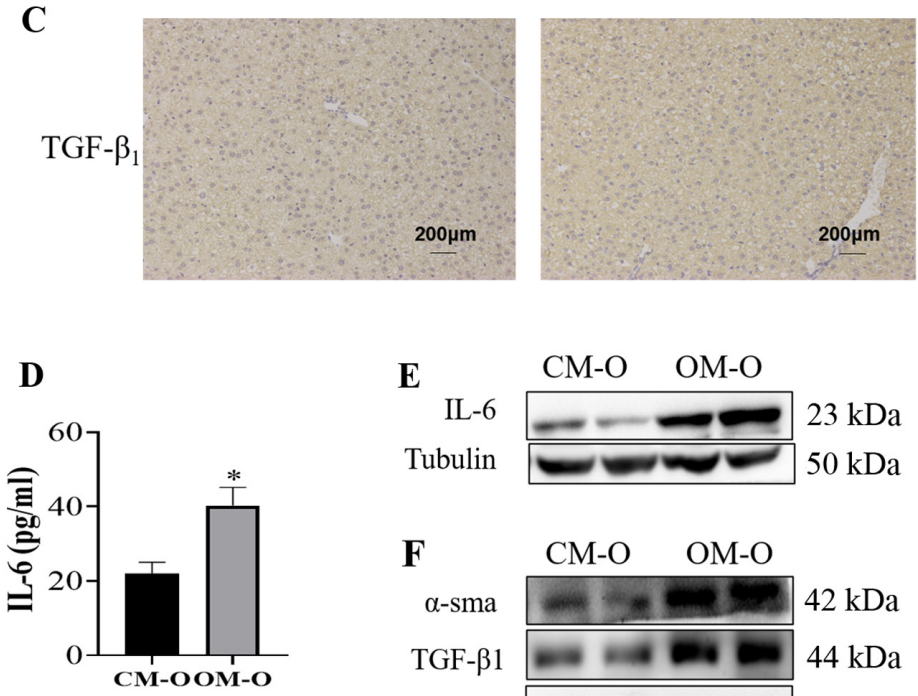

E

Tubulin

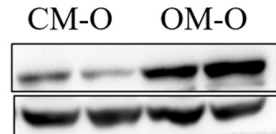

$\mathbf{F}$

$\alpha-\operatorname{sma}$

TGF- $\beta 1$

Tubulin

\section{$\mathrm{CM}-\mathrm{O} \quad \mathrm{OM}-\mathrm{O}$}

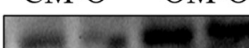

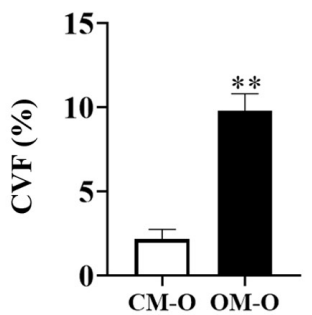
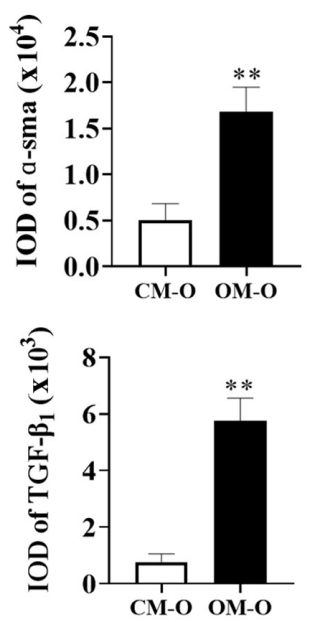

Figure 4. NAFLD is associated with liver fibrosis. (A) Representative image of Masson staining in liver sections ( $\mathrm{n}=3 / \mathrm{group}$; magnification, $\mathrm{x} 200$ ). (B and C) Representative pictures of $\alpha$-sma and TGF- $\beta 1$ immunohistochemical staining, respectively in liver are shown ( $\mathrm{n}=3 / \mathrm{group}$; magnification, $\mathrm{x} 200$ ). (D) Serum IL-6 was detected in offspring at 17 weeks ( $\mathrm{n}=6 /$ group). ( $\mathrm{E}$ and F) Representative blots of IL-6, $\alpha$-sma and TGF- $\beta 1$ expression in offspring mice liver are shown ( $\mathrm{n}=3$ /group). (G) Expression of IL-6, $\alpha$-sma and TGF- $\beta 1$ in 17 weeks old offspring by WB ( $\mathrm{n}=3 /$ group). ${ }^{*} \mathrm{P}<0.05,{ }^{* * *} \mathrm{P}<0.01$. NAFLD, non-alcoholic fatty liver disease; CM-O, offspring of chow mother; OM-O, offspring of obese mother; IL-6, interleukin-6; $\alpha$-sma, alpha-smooth muscle actin; TGF- $\beta 1$, transforming growth factor beta 1; IOD, integral optical density; CVF, Collagenvolume fraction.

in the liver in OM-O group compared with the CM-O group and that $\alpha$-sma expression significantly increased in the OM-O group compared with the CM-O group $(\mathrm{P}<0.05)$ (Fig. 4E and $\mathrm{G})$. These results indicated that while maternal obesity led 
to the development of NAFLD in the offspring, it was accompanied by an increase in liver fibrosis.

\section{Discussion}

NAFLD global prevalence is increasing year on year and it is widely known that maternal obesity is closely related to the occurrence of NAFLD in the offspring (27). Cantoral et al (28) found that maternal overweight before pregnancy was closely related to liver fat content of offspring in a cohort study. Another study demonstrated that intervention of maternal rats with high-fat diet during pregnancy or early pregnancy can lead to severe liver steatosis and NAFLD risk in offspring (29). In the present study, an obese female mouse model was established via a high-fat diet and it was found that the mice of the OM-O group were more likely to form NAFLD than the CM-O group. By investigation of biochemical indicators and tissue sections of the offspring in the present study, it was revealed that TC, TG and LDL of the OM-O group increased, while the HDL levels showed a downward trend compared with the CM-O group. Notably, the present study by performing $\mathrm{HE}$ and oil red staining demonstrated that the OM-O group had more lipid droplets than the CM-O group. Previous epidemiological studies have reported that maternal obesity is an independent risk factor for the development of NAFLD in offspring $(27,30,31)$. However, the present study did not provide sufficient data to prove that the degree of maternal obesity was positively associated with the severity of NAFLD in the offspring. It is likely that this may be caused by some uncontrollable factors regarding the maternal generation in epidemiological studies, such as dietary structure, circadian rhythm etc. The findings of the present study demonstrated that maternal obesity is one of the susceptibility factors for NAFLD in the offspring, but it cannot yet be stated definitively that the higher the obesity of the maternal generation, the more severe the occurrence of NAFLD in the offspring. However, the results of the present study are sufficient to provide a warning to mothers that obesity serves an important role in the occurrence and exacerbation of NAFLD in the offspring.

A study demonstrated that autophagy may serve a role in NAFLD formation in offspring (32). Lipophagy (a type of selective autophagy) mainly involves the selective degradation of cytoplasmic lipid droplets, so hepatocyte autophagy is currently considered a defense mechanism in the prevention of NAFLD (33). On the contrary, a study has demonstrated that excessive triglycerides and free fatty acids in NAFLD caused lipid effects, such as insulin resistance and oxidative stress to inhibit autophagy activity and then a decrease in autophagy activity further aggravates the production of NAFLD (34). Yet other studies have reported that using rapamycin and carbamazepine promotes autophagy to reduce NAFLD occurrence $(35,36)$. The present study demonstrated that beclin-1, ATG3, ATG5, ATG12 and LC3B expression in OM-O livers was lower than mice of the CM-O group, while the expression of P62 was increased, which indicated that OM-O livers were inhibited during this period. In the present study, the livers of the OM-O group had severe lipid droplet deposition and a large increase in serum IL-6, fully indicating that the decrease in autophagy was aggravated to some extent, which was confirmed by previous studies (37-39). Liu et al (40) found that in SD rats fed a high-fat diet, the early stage of steatosis revealed higher levels of LC3 II/I, lower p62 and higher fat cholesterol in the late stage, suggesting that autophagy is usually activated in the early stages of steatosis, but then blocked in at a late stage. The aforementioned results indicated that autophagy is more likely to be blocked in the late obese generation, and the decrease of autophagy level is more likely to increase the susceptibility to NAFLD in the offspring of an obese mother.

The present study also demonstrated that the liver NAFLD formation induced by maternal obesity may be related to the changes of autophagy upstream signal AMPK and mTOR. Recently, a liver-specific genetic study confirmed the important role of AMPK in HFD induced hepatic steatosis in NAFLD mice (41). While, a large number of studies have also shown that the activation of AMPK signal pathway can reduce the deposition of lipid droplets and the occurrence of NAFLD. Zhou et al (42) found that a low dose of aspirin may reduce lipid droplet deposition via AMPK activation in the offspring of obese mothers. Devarajan et al (32) also reported that the occurrence of NAFLD in offspring with energy limitation during the perinatal period was related to AMPK changes. AMPK is the main positive regulator of autophagy and MTOR is the key negative regulator of autophagy (43). Soto-Avellaneda et al (44) demonstrated that statins can alter the synthesis of cholesterol free fatty acids by inhibiting the activation of mTOR site and autophagy, hence reducing the deposition of lipids in non-adipose tissues, such as the liver. The aforementioned results were consistent with the findings of the present study and that the change of AMPK-mTOR pathway may promote the formation of NAFLD in obese children.

NAFLD is a type of systemic liver disease, which ranges from simple steatosis to steatohepatitis, and can progress to fibrosis, cirrhosis, and finally liver cancer in some cases (45). Previous studies have revealed that the inflammatory factor, IL- 6 and fibrosis indicators, TGF- $\beta 1$ and $\alpha$-sma, are highly expressed in NAFLD (46-48). In the process of liver fibrosis, apart from TGF- $\beta 1$ and $\alpha$-sma, as an important indicator of liver fibrosis, serum IL-6 also has been widely reported (49). Wei et al (50) established a rat model of liver fiber by carbon tetrachloride. Through the detection of serum IL-6, it was found that the content of serum IL- 6 in the CCL4 group was significantly higher compared with that in the wild group, but following the addition of plumbagin, the hepatic fibrosis induced by CCL4 was significantly improved and serum IL-6 was significantly decreased (50). A study by Al-Hashem et al (51) treated mice with hepatic fibrosis with metformin and it was revealed that the liver fibrosis of mice treated with metformin was significantly improved and the level of serum IL-6 was significantly decreased. A large number of clinical studies have found that compared with healthy subjects, the content of serum IL-6 in patients with NAFLD is significantly increased $(52,53)$. Hence, it was hypothesized that the level of IL- 6 may be used as an important biological indicator of liver fibrosis. In liver fibrosis, IL-6 is not studied as a single indicator (54), hence in the present study liver $\alpha$-SMA and TGF- $\beta 1$ were studied as well. In the present study, in order to verify whether maternal obesity is responsible for fibrosis change in the offspring, ELISA and 
western blotting was used to measure IL- $6, \alpha$-SMA and TGF- $\beta 1$ in the offspring and it was demonstrated that with the continuous development of NAFLD at 17 weeks, the expression levels of IL- 6 , TGF- $\beta 1$, and $\alpha$-sma in the livers of the OM-O group was significantly higher than CM-O mice. Mouralidarane et al (55) published similar findings regarding liver fibrosis in the offspring of obese female mice at different time points.

In addition, there is growing evidence that innate immunity is the driving force behind the progression of NAFLD $(56,57)$. As an important immune organ, the liver contains a coordinated network of innate immune cells, such as Kupffer cell (KCs), dendritic cell (DC), lymphocytes, neutrophils and mast cells. Mouralidarane et al established an obese maternal mouse model to induce hyperlipidemia in their offspring during pregnancy and found that the liver phenotype of their offspring deteriorated significantly in adulthood. In addition, it was also found the number of $\mathrm{KCs}$ in the innate immune system of the liver increased but its cell phagocytosis decreased, is not conducive to liver lipid clearance (55). KCs can release a large number of cytokines and chemokines, such as tumor necrosis factor (TNF)- $\alpha$, IL-6 etc. further promoting hepatocyte production and complement protein release and a large number of complement and inflammatory factors lead to liver fat deformation and inflammatory changes (58). The expression of inflammatory cytokines IL-6, TNF- $\alpha$ and TGF- $\beta 1$ has also been demonstrated to change with the regulation of the immune system (59-61). Based on the finding of previous studies, it was hypothesized that innate immunity serves an important role in the formation and development of NAFLD, while inflammatory factors may be regulated by the innate immune response, hence playing an important role in the development of disease. The findings of previous studies indicated that the occurrence of NAFLD susceptibility in the offspring due to maternal obesity may be related to innate immunity. Future studies need to study this in more detail.

The study has certain limitations. Only the changes of the OM-O and CM-O groups were observed at 17 weeks, meaning that the changes of offspring mice beyond this time was not assessed. At the same time, with regard to the changes of liver inflammation in mice, only the changes of IL-6 were assessed, despite there being other inflammatory factors that may serve a role. Future studies should assess the relationship between autophagy and NAFLD in obese offspring at different times,and elucidate the role of different inflammatory factors in NAFLD obese offspring.

In conclusion, the present study revealed the phenomenon of altered autophagy in a NAFLD mouse model of offspring caused by maternal obesity and in addition, demonstrated the role of AMPK/mTOR signal in this process. Changes in autophagy may serve a key role in the development of maternal obesity and NAFLD in offspring, which may lead to the early prevention of NAFLD. Autophagy may be used as a new target to prevent maternal obesity causing NAFLD in the offspring.

\section{Acknowledgements}

Not applicable.

\section{Funding}

This work was supported by Natural Science Foundation of Zhejiang Province (grant no. LY18H040014) and Natural Science Foundation of Anhui Province (grant no. KJ2019A0353).

\section{Availability of data and materials}

The datasets used and/or analyzed during the current study are available from the corresponding author on reasonable request.

\section{Authors' contributions}

YT and GJ designed the study. SH, FZ, XH, PY, FS and KX performed the experiments. SH, FZ, JS and ZY analyzed the data. SH and FZ wrote the manuscript. SH and FZ confirm the authenticity of all the raw data. All authors have read and approved the final manuscript.

\section{Ethics approval and consent to participate}

The present study was approved by the Animal Experiments and Animal Experiment Ethics Committee of The Second Affiliated Hospital of Jiaxing University, Jiaxing Second Hospital (Jiaxing, China; approval no. jxey2017002).

\section{Patient consent for publication}

Not applicable.

\section{Competing interests}

The authors declare that they have no competing interests.

\section{References}

1. Byrne CD and Targher G: NAFLD: A multisystem disease. J Hepatol 62 (Suppl 1): S47-S64, 2015.

2. Fan JG, Kim SU and Wong VJJ: New trends on obesity and NAFLD in asia. J Hepatol 67: 862-873, 2017.

3. Estes C, Anstee QM, Arias-Loste MT, Bantel H, Bellentani S, Caballeria J, Colombo M, Craxi A, Crespo J, Day CP, et al: Modeling NAFLD disease burden in China, France, Germany, Italy, Japan, Spain, United Kingdom, and United States for the period 2016-2030. J Hepatol 69: 896-904, 2018.

4. Ayonrinde OT, Adams LA, Mori TA, Beilin LJ, de Klerk N, Pennell CE, White S and Olynyk JK: Sex differences between parental pregnancy characteristics and nonalcoholic fatty liver disease in adolescents. Hepatology 67: 108-122, 2018.

5. Patel S, Lawlor DA, Callaway M, Macdonald-Wallis C, Sattar N and Fraser A: Association of maternal diabetes/glycosuria and pre-pregnancy body mass index with offspring indicators of non-alcoholic fatty liver disease. BMC Pediatr 16: 47, 2016.

6. Pereira TJ, Fonseca MA, Campbell KE, Moyce BL, Cole LK, Hatch GM, Doucette CA, Klein J, Aliani M and Dolinsky VW: Maternal obesity characterized by gestational diabetes increases the susceptibility of rat offspring to hepatic steatosis via a disrupted liver metabolome. J Physiol 15: 3181-3197, 2015.

7. Bouanane S, Merzouk H, Benkalfat NB, Soulimane N, Merzouk SA, Gresti J, Tessier C and Narce M: Hepatic and very low-density lipoprotein fatty acids in obese offspring of overfed dams. Metabolism 59: 1701-1709, 2010.

8. Liou CJ, Wu SJ, Shen SC, Chen LC, Chen YL and Huang WC: Phloretin ameliorates hepatic steatosis through regulation of lipogenesis and Sirt1/AMPK signaling in obese mice. Cell Biosci 10: 114, 2020. 
9. Wilson RB, Chen YJ, Sutherland BG, Sawyez CG, Zhang R, Woolnough T, Hetherington AM, Peters KM, Patel K, Kennelly JP, et al: The marine compound and elongation factor $1 \mathrm{~A} 1$ inhibitor, didemnin B, provides benefit in western diet-induced non-alcoholic fatty liver disease. Pharmacol Res 161: 105208, 2020.

10. Borengasser SJ, Lau F, Kang P, Blackburn ML, Ronis MJ, Badger TM and Shankar K: Maternal obesity during gestation impairs fatty acid oxidation and mitochondrial SIRT3 expression in rat offspring at weaning. PLoS One 6: e24068, 2011.

11. Wankhade UD, Zhong Y, Kang P, Alfaro M, Chintapalli SV, Thakali KM and Shankar K: Enhanced offspring predisposition to steatohepatitis with maternal high-fat diet is associated with epigenetic and microbiome alterations. PLoS One 12: e0175675, 2017.

12. Yamaguchi R, Nakagawa Y, Liu YJ, Fujisawa Y, Sai S Nagata E, Sano S, Satake E, Matsushita R, Nakanishi T, et al: Effects of maternal high-fat diet on serum lipid concentration and expression of peroxisomal proliferator-activated receptors in the early life of rat offspring. Horm Metab Res 42: 821-825, 2010.

13. Paul HA, Bomhof MR, Vogel HJ and Reimer RA: Diet-induced changes in maternal gut microbiota and metabolomic profiles influence programming of offspring obesity risk in rats. Sci Rep 6: 20683, 2016.

14. Glick D, Barth S and Macleod KF: Autophagy: Cellular and molecular mechanisms. J Pathol 221: 3-12, 2010.

15. Levine B and Kroemer G: Biological functions of autophagy genes: A disease perspective. Cell 176: 11-42, 2019.

16. Settembre C, De Cegli R, Mansueto G, Saha PK, Vetrini F, Visvikis O, Huynh T, Carissimo A, Palmer D, Klisch TJ, et al: TFEB controls cellular lipid metabolism through a starvation-induced autoregulatory loop. Nat Cell Biol 15: 647-658, 2013.

17. Zhang H, Yan S, Khambu B, Ma F, Li Y, Chen X, Martina JA, Puertollano R, Li Y, Chalasani N and Yin XM: Dynamic MTORC1-TFEB feedback signaling regulates hepatic autophagy, steatosis and liver injury in long-term nutrient oversupply. Autophagy 14: 1779-1795, 2018.

18. Li S, Dou X, Ning H, Song Q, Wei W, Zhang X, Shen C, Li J, Sun $C$ and Song Z: Sirtuin 3 acts as a negative regulator of autophagy dictating hepatocyte susceptibility to lipotoxicity. Hepatology 66: 936-952, 2017.

19. Chao X, Wang H, Jaeschke H and Ding WX: Role and mechanisms of autophagy in acetaminophen-induced liver injury. Liver Int 38: 1363-1374, 2018

20. Chu Q, Zhang S, Chen M, Han W, Jia R, Chen W and Zheng X: Cherry anthocyanins regulate NAFLD by promoting autophagy pathway. Oxid Med Cell Longev 2019: 4825949, 2019.

21. Livak KJ and Schmittgen TD: Analysis of relative gene expression data using real-time quantitative PCR and the 2(-Delta Delta C(T)) method. Methods 25: 402-408, 2001.

22. Zhou B, Liu J, Kang R, Klionsky DJ, Kroemer G and Tang D: Ferroptosis is a type of autophagy-dependent cell death. Semin Cancer Biol 66: 89-100, 2020.

23. Warnes G: Flow cytometric assays for the study of autophagy. Methods 82: 21-28,2015.

24. Kim J, Kundu M, Viollet B and Guan KL: AMPK and mTOR regulate autophagy through direct phosphorylation of Ulk1. Nat Cell Biol 13: 132-141, 2011

25. Jia J, Abudu YP, Claude-Taupin A, Gu Y, Kumar S, Choi SW Peters R, Mudd MH, Allers L, Salemi M, et al: Galectins control MTOR and AMPK in response to lysosomal damage to induce autophagy. Autophagy 15: 169-171, 2019.

26. Zhou Y, Wu R, Cai FF, Zhou WJ, Lu YY, Zhang H, Chen QL and Su SB: Xiaoyaosan decoction alleviated rat liver fibrosis via the TGF $3 /$ Smad and Akt/FoxO3 signaling pathways based on network pharmacology analysis. J Ethnopharmacol 264: 113021, 2021.

27. Ayonrinde OT, Oddy WH, Adams LA, Mori TA, Beilin LJ, de Klerk $\mathrm{N}$ and Olynyk JK: Infant nutrition and maternal obesity influence the risk of non-alcoholic fatty liver disease in adolescents. J Hepatol 67: 568-576, 2017.

28. Cantoral A, Montoya A, Luna-Villa L, Roldán-Valadez EA, Hernández-Ávila M, Kershenobich D, Perng W, Peterson KE, Hu H, Rivera JA and Téllez-Rojo MM: Overweight and obesity status from the prenatal period to adolescence and its association with non-alcoholic fatty liver disease in young adults: Cohort study. BJOG 127: 1200-1209, 2020 .
29. Zhou Y, Peng H, Xu H, Li J, Golovko M, Cheng H, Lynch EC, Liu L, McCauley N, Kennedy L, et al: Maternal diet intervention before pregnancy primes offspring lipid metabolism in liver. Lab Invest 100: 553-569, 2020

30. Alisi A and Vajro P: Pre-natal and post-natal environment monitoring to prevent non-alcoholic fatty liver disease development. J Hepatol 67: 451-453, 2017.

31. Simpson J, Smith AD, Fraser A, Sattar N, Callaway M, Lindsay RS, Lawlor DA and Nelson SM: Cord blood adipokines and lipids and adolescent nonalcoholic fatty liver disease. J Clin Endocrinol Metab 101: 4661-4668, 2016.

32. Devarajan A, Rajasekaran NS, Valburg C, Ganapathy E, Bindra S and Freije WA: Maternal perinatal calorie restriction temporally regulates the hepatic autophagy and redox status in male rat. Free Radic Biol Med 130: 592-600, 2019.

33. Singh R, Kaushik S, Wang Y, Xiang Y, Novak I, Komatsu M, Tanaka K, Cuervo AM and Czaja MJ: Autophagy regulates lipid metabolism. Nature 458: 1131-1135, 2009.

34. Martinez-Lopez N and Singh R: Autophagy and lipid droplets in the liver. Annual Rev Nutr 35: 215-237, 2015.

35. González-Rodríguez A, Mayoral R, Agra N, Valdecantos MP, Pardo V, Miquilena-Colina ME, Vargas-Castrillón J, Lo Iacono O, Corazzari M, Fimia GM, et al: Impaired autophagic flux is associated with increased endoplasmic reticulum stress during the development of NAFLD. Cell Death Dis 5: e1179, 2014

36. Lin CW, Zhang H, Li M, Xiong X, Chen X, Chen X, Dong XC and Yin XM: Pharmacological promotion of autophagy alleviates steatosis and injury in alcoholic and non-alcoholic fatty liver conditions in mice. J Hepatol 58: 993-999, 2013.

37. Guo R, Nair S, Zhang Y and Ren J: Adiponectin deficiency rescues high-fat diet-induced hepatic injury, apoptosis and autophagy loss despite persistent steatosis. Int J Obes (Lond) 41 1403-1412, 2017.

38. Lu W, Mei J, Yang J, Wu Z, Liu J, Miao P, Chen Y, Wen Z, Zhao Z, Kong H, et al: ApoE deficiency promotes non-alcoholic fatty liver disease in mice via impeding AMPK/mTOR mediated autophagy. Life Sci 252: 117601,2020.

39. Lee DH, Park SH, Ahn J, Hong SP, Lee E, Jang YJ, Ha TY, Huh YH, Ha SY, Jeon TI and Jung CH: Mir214-3p and Hnf4a/Hnf4 $\alpha$ reciprocally regulate Ulk1 expression and autophagy in nonalcoholic hepatic steatosis. Autophagy, 2020 (Epub ahead of print)

40. Liu C, Liu L, Zhu HD, Sheng JQ, Wu XL, He XX, Tian DA, Liao JZ and Li PY: Celecoxib alleviates nonalcoholic fatty liver disease by restoring autophagic flux. Sci Rep 8: 4108, 2018.

41. Garcia D, Hellberg K, Chaix A, Wallace M, Herzig S, Badur MG, Lin T, Shokhirev MN, Pinto AFM, Ross DS, et al: Genetic liver-specific AMPK activation protects against diet-induced obesity and NAFLD. Cell Rep 26: 192-208.e6, 2019.

42. Zhou Y, Peng H, Liu Z, Zhang KK, Jendrusch C, Drake M, Hao Y and Xie L: Sex-associated preventive effects of low-dose aspirin on obesity and non-alcoholic fatty liver disease in mouse offspring with over-nutrition in utero. Lab Invest 99: 244-259, 2019.

43. Inoki K, Kim J and Guan KL: AMPK and mTOR in cellular energy homeostasis and drug targets. Annu Rev Pharmacol Toxicol 52: 381-400, 2012.

44. Soto-Avellaneda A and Morrison BE: Signaling and other functions of lipids in autophagy: A review. Lipids Health Dis 19: 214 2020.

45. Rinella ME and Sanyal AJ: Management of NAFLD: A stage-based approach. Nat Rev Gastroenterol Hepatol 13: 196-205, 2016

46. Fang C, Cai X, Hayashi S, Hao S, Sakiyama H, Wang X, Yang Q, Akira S, Nishiguchi S, Fujiwara N, et al: Caffeine-stimulated muscle IL-6 mediates alleviation of non-alcoholic fatty liver disease. Biochim Biophys Acta Mol Cell Biol Lipids 1864: 271-280, 2019.

47. Hart KM, Fabre T, Sciurba JC, Gieseck RL, Borthwick LA, Vannella KM, Acciani TH, de Queiroz Prado R, Thompson RW, White $\mathrm{S}$, et al: Type 2 immunity is protective in metabolic disease but exacerbates NAFLD collaboratively with TGF- $\beta$. Sci Transl Med 9: eaal3694, 2017.

48. Chen P, Luo Q, Huang C, Gao Q, Li L, Chen J, Chen B, Liu W, Zeng $\mathrm{W}$ and Chen Z: Pathogenesis of non-alcoholic fatty liver disease mediated by YAP. Hepatol Int 12: 26-36, 2018

49. Stojsavljević S, Gomerčić Palčić M, Virović Jukić L, Smirčić Duvnjak L and Duvnjak M: Adipokines and proinflammatory cytokines, the key mediators in the pathogenesis of nonalcoholic fatty liver disease. World J Gastroenterol 20: 18070-18091, 2014. 
50. Wei Y, Huang M, Liu X, Yuan Z, Peng Y, Huang Z and Zhao T: Anti-fibrotic effect of plumbagin on CCl4-lesioned rats. Cell Physiol Biochem 35: 1599-1608, 2015.

51. Al-Hashem F, Al-Humayed S, Amin SN, Kamar SS, Mansy SS, Hassan S, Abdel-Salam LO, Ellatif MA, Alfaifi M, Haidara MA and Al-Ani B: Metformin inhibits mTOR-HIF-1 $\alpha$ axis and profibrogenic and inflammatory biomarkers in thioacetamideinduced hepatic tissue alterations. J Cell Physiol 234: 9328-9337, 2018.

52. Haukeland JW, Damås JK, Konopski Z, Løberg EM, Haaland T, Goverud I, Torjesen PA, Birkeland K, Bjøro K and Aukrust P: Systemic inflammation in nonalcoholic fatty liver disease is characterized by elevated levels of CCL2. J Hepato 44: 1167-1174, 2006.

53. García-Galiano D, Sánchez-Garrido MA, Espejo I, Montero JL, Costán G, Marchal T, Membrives A, Gallardo-Valverde JM, Muñoz-Castañeda JR, Arévalo E, et al: IL-6 and IGF-1 are independent prognostic factors of liver steatosis and non-alcoholic steatohepatitis in morbidly obese patients 17: 493-503, 2007.

54. Mridha AR, Wree A, Robertson AAB, Yeh MM, Johnson CD Van Rooyen DM, Haczeyni F, Teoh NC, Savard C, Ioannou GN, et al: NLRP3 inflammasome blockade reduces liver inflammation and fibrosis in experimental NASH in mice. J Hepatol 66: 1037-1046, 2017.

55. Mouralidarane A, Soeda J, Visconti-Pugmire C, Samuelsson AM, Pombo J, Maragkoudaki X, Butt A, Saraswati R, Novelli M, Fusai G, et al: Maternal obesity programs offspring nonalcoholic fatty liver disease by innate immune dysfunction in mice. Hepatology 58: 128-138, 2013.
56. Schuster S, Cabrera D, Arrese M and Feldstein AE: Triggering and resolution of inflammation in NASH. Nat Rev Gastroenterol Hepatol 15: 349-364, 2018.

57. Gao B, Jeong WI and Tian Z: Liver: An organ with predominant innate immunity. Hepatology 47: 729-736, 2008.

58. Arrese M, Cabrera D, Kalergis AM and Feldstein AE: Innate Immunity and Inflammation in NAFLD/NASH. Dig Dis Sci 61: 1294-1303, 2016

59. Yu Y,Liu Y, An W, Song J,Zhang Y and Zhao X: STING-mediated inflammation in Kupffer cells contributes to progression of nonalcoholic steatohepatitis. J Clin Invest 129: 546-555, 2019.

60. Hurrell BP, Galle-Treger L, Jahani PS, Howard E, Helou DG, Banie H, Soroosh P and Akbari O: TNFR2 Signaling enhances ILC2 survival, function, and induction of airway hyperreactivity. Cell Rep 29: 4509-4524.e5. 2019.

61. Fang W, Deng Z, Benadjaoud F, Yang C and Shi GP: Cathepsin B deficiency ameliorates liver lipid deposition, inflammatory cell infiltration, and fibrosis after diet-induced nonalcoholic steatohepatitis. Transl Res 222: 28-40, 2020.

This work is licensed under a Creative Commons Attribution-NonCommercial-NoDerivatives 4.0 International (CC BY-NC-ND 4.0) License. 